\title{
Natural Leaders
}

\section{Some interlocutors elicit greater convergence across conversations and across characteristics}

\author{
Uriel Cohen Priva $\quad$ Chelsea Sanker
}

\begin{abstract}
Are there natural followers in human communication, speakers who consistently converge more than others? Similarly, are there natural leaders, speakers with whom others converge more? Are such tendencies consistent across different linguistic characteristics? We use the Switchboard Corpus, a large collection of telephone conversations, to perform a large-scale study of convergence of speakers in multiple conversations with different interlocutors, across six linguistic characteristics. Having data from each speaker in several conversations makes it possible to investigate whether there are individual differences in likelihood to converge, among speakers (more or less likely to converge), and among interlocutors (more or less likely to elicit convergence). We only find evidence for individual differences by interlocutor, not by speaker; this shows that there are natural leaders, who elicit greater degrees of convergence than others, across different characteristics and different conversations. The absence of a similar finding for natural followers, speakers who converge more than others, suggests that the role of social aspects in mediating convergence is stronger than that of putative individual differences in propensity to converge, or that such propensities are characteristic-specific.
\end{abstract}

\section{Introduction}

Are there individuals who are more likely than others to change their performance such that it is more similar to their interlocutors'? Are there interlocutors who are more likely than others to cause such shifts? Convergence, in which individuals' behaviors become more similar to their interlocutors', has been demonstrated in many characteristics, both linguistic and non-linguistic. ${ }^{1}$

\footnotetext{
${ }^{1}$ Convergence, as thus defined, only includes alignment between individuals, and not other sorts of interlocutor accommodation. Convergence can be measured with two basic types of comparisons: overall increased similarity
} 
Many convergence studies find variation in degree of convergence across participants. This variation is sometimes attributed to individual differences in tendency to converge, which could indicate a broad cognitive trait producing these differences, but little work has tested whether tendencies are consistent for an individual across characteristics or across tasks. The existence of individual differences in convergence is called into question by several studies that have looked for individual consistency but found weak evidence or no evidence (Pardo et al., 2012; Sanker, 2015; Weise \& Levitan, 2018; Pardo et al., 2018). Less work has addressed differences in the degree of convergence elicited by particular interlocutors. Consistency of convergence by speaker or by interlocutor across characteristics would suggest that social mediation of convergence is affecting different characteristics in the same way.

In this paper, we present a meta-analysis of six convergence studies to probe factors that influence convergence: the characteristic measured, the speaker, the interlocutor, and the particular conversation. The individual convergence studies examine six speech characteristics of different sorts, measured in the same conversational recordings: F0 median, F0 variance, speech rate, uh:um ratio, lexical information rate, and sentential conjunction. Even when the characteristics are normalized, there is variability in the degree of convergence across different characteristics Nevertheless, the meta-analysis finds consistency in convergence by interlocutor, indicating that there are external social factors at a high level that influence convergence in different linguistic characteristics similarly; some speakers elicit more convergence than others do. However, we do not find consistency by speaker, which suggests that there might not be any individual-specific cognitive trait which predisposes some speakers to converge more than others. Individual tendencies in convergence appear only within particular characteristics.

\subsection{Individual differences in convergent traits}

Degree of convergence may be influenced by traits of the participants, as some personal traits have been found to be significant predictors of degree of convergent behavior, such as higher openness and attentional focus scores (Yu et al., 2013) or higher social desirability scores (Natale, 1975). Yu et al. (2013) regard this variation as evidence for individual differences in language processing. Some work has demonstrated individual differences in other linguistic behaviors, for example, categorical perception (Kong \& Edwards, 2016) or lexical bias (Ishida et al., 2016), so it would be reasonable to expect similar individual differences in convergence. However, the convergence studies on individual tendencies did not retest individual to establish their consis-

of a speaker to an interlocutor or model talker (e.g. Goldinger, 1998; Pardo et al., 2017; Babel, 2009) and synchrony over time (e.g. Schweitzer \& Lewandowski, 2013; Levitan \& Hirschberg, 2011). Our study only examines the former. 
tency, so interpretations are at risk of a fundamental attribution error. Many studies implicitly or explicitly assume that variation in convergence reflects characteristics of individuals, but only a few studies have tested the same individuals in multiple conversations or experimental tasks. Individuals do exhibit some consistent tendencies in convergence in the same or very similar tasks, when measured in the same characteristic (Sanker, 2015; Tamminga et al., 2018), but these tendencies are much weaker across more dissimilar tasks such as shadowing and conversation (Pardo et al., 2018). In conversations, tendencies of an individual in producing convergence cannot be distinguished from tendencies of an individual to elicit convergence, which could confound the results when each speaker participates in a single conversation.

Is there a cognitive trait that differs across individuals, producing variation in convergence? One possible source of individual variation in convergence is attention, particularly as mediated by social investment (Yu et al., 2013). In addition to social factors predicting convergence, direct manipulations of attention also show effects on convergence; speakers converge less when they have a larger cognitive load (Abel \& Babel, 2017; Heath, 2017). Related to attention, differences in memory would be a possible candidate for individual variation, though Yu et al. (2013) found no relationship between working memory and convergence. In any case, interpreting individual tendencies first depends on demonstrating that robust replicable individual tendencies exist.

If there is a broad cognitive trait driving individual variation in convergence, its effects should be consistent across different characteristics within which convergence is measured (Weise \& Levitan, 2018; Cohen Priva \& Sanker, 2018); an individual pattern of convergence in one characteristic should be predictive of that speaker's convergence in other characteristics. Even if absolute convergence in each characteristic differs, the relative convergence in each characteristic should correlate across individuals. However, there is little work that compares convergence by each individual across characteristics and most studies that have looked for such patterns found no effect (Bilous \& Krauss, 1988; Pardo et al., 2012; Sanker, 2015; Weise \& Levitan, 2018), though the null result could potentially be attributed to the rather small number of participants in most of these studies.

Some theoretical accounts explicitly predict a correlation of convergence across characteristics, based on alignment at one level facilitating alignment at other levels (Pickering \& Garrod, 2004, pp. 174-175). This theoretical alignment might be observed in neural synchronization, which has been found to increase along with behavioral synchrony (Yun et al., 2012). Phonetic convergence likely reflects an intrinsic link between perception and production, such that incoming speech automatically activates motor plans that are subsequently reflected in production (Pickering \& Garrod, 2013). Thus, effects should be consistent across tasks, and different phonetic characteristics should exhibit the same influences, though lexical and syntactic characteristics might 
behave differently. Pardo et al. (2018) argue that such a perception-production link cannot be automatic, because it varies based on context, resulting in different convergence behavior of the same speaker in different tasks.

\subsection{Social mediation}

Variation in degree of convergence might be due to social factors rather than or in addition to individual cognitive differences. Some individuals might elicit more or less convergence, either based on their behavior, or based on what the listeners know or think they know about them; previous work has demonstrated some such effects (Babel, 2009; Yu et al., 2011; Pardo et al., 2017). Many studies have found that aspects of the interlocutor or model talker's perceived identity or the speaker's perspective towards that identity can influence convergence. Factors that have been found to influence convergence include gender (e.g. Bilous \& Krauss, 1988; Namy et al., 2002), native language (Kim et al., 2011), perceived standardness of the model talker's dialect (Weatherholtz et al., 2014), interlocutor status (Gregory \& Webster, 1996), and attitude towards a model talker (e.g. Babel, 2010; Yu et al., 2011). The observed effects of these social factors on convergence are not always consistent across different studies.

Some variation across interlocutors may be due to differences in amount of exposure to their speech. More exposure to a construction or a lexical item increases the probability that a subject will subsequently produce that item (Kaschak et al., 2011; Oben \& Brône, 2016), though cumulative effects in phonetic convergence are less clear (e.g. Babel, 2012; Gijssels et al., 2016). In conversational tasks, different amounts of speech produced by each individual would produce different levels of exposure and might result in different degrees of convergence; talkative individuals might elicit more convergence than others do. However, variation in the degree of convergence elicited by different interlocutors or model talkers has been found even in tasks with equal exposure to each voice (e.g. Pardo et al., 2017; Hwang \& Chun, 2018), indicating that there must be factors other than just exposure.

Social mediation via the speaker's opinion of the interlocutor is a likely source of interlocutor effects in convergence. In studies that specifically manipulate the subject's opinion of the model talker, a more positive opinion predicted greater convergence (Babel, 2010; Yu et al., 2011). In conversations in which subjects were allowed to develop opinions naturally, liking of the interlocutor also predicted greater convergence (Sanker, 2015). Greater perceived attractiveness of a voice is also predictive of how much convergence it elicits (Babel et al., 2014). Communication Accommodation Theory proposes that speakers use convergence and divergence to manipulate social distance from interlocutors (Giles et al., 1973, 1991). However, socially mediated convergence 
does not necessarily need to be done consciously; interlocutor effects may result from greater weight given to input from speakers who are viewed more positively (Chartrand \& Bargh, 1999). One possible mechanism is offered by Jiang et al. (2015), based on interpersonal neural synchrony; the speakers whose partners synchronize more with them tend to perceived as "leaders", based on a range of skills in communication and reasoning.

Degree of convergence is also predicted by aspects of the interaction and the relationship between the interlocutors (e.g. Gregory \& Webster, 1996; Pardo et al., 2012; Bane et al., 2010). If variation is driven by situational or interactional factors that increase convergence via broad mechanisms such as increased attention, there should be consistency between participants within the same conversation and across different characteristics within that conversation. Little work has systematically tested whether the convergence exhibited by a conversing pair in one characteristic was predictive of their convergence in other characteristics within the same interaction. In a comparison across a small number of conversations, Sanker (2015) found only a weak trend towards positive correlations across characteristics. With a larger dataset, Weise \& Levitan (2018) found no correlation in convergence across characteristics. Cohen Priva \& Sanker (2018) found a correlation only between closely related characteristics: F0 median and F0 variability.

Many studies include each individual only in a single interaction, and thus cannot separate out the individual contributions of each speaker to convergence in a conversation, so tendencies by speaker, by interlocutor, or by conversation would all produce the same pattern of variability. A study that includes each individual in multiple interactions with different partners makes it possible to separate speaker, interlocutor, and conversation as possible factors with distinct tendencies in convergence.

\section{Methods Overview}

\subsection{Corpus}

The data for this study is the Switchboard Corpus (Godfrey \& Holliman, 1997), a large collection of telephone conversations. Each speaker was randomly paired with other speakers and given a topic for each conversation, providing a large corpus of natural speech data for many speakers in similar conversations with several different partners. Recordings include associated speaker identification data that can be used to compare all instances of that speaker in different conversations. Each side of the conversation is a distinct recording, so measurements can reliably be taken for each speaker separately. 
Each conversation has associated information quantifying the clarity of the recording. To ensure reliable acoustic measurements (F0 median and variance), calls were omitted if they had high levels of background noise, echoing, or other issues, as indicated in their clarity rating. This resulted in a total of 464 speakers in the data used for acoustic characteristics. For measurements that did not depend on acoustic form, no conversations were omitted, so these measurements were based on 518 speakers. Conversations took 6:20 minutes on average (the median was 5 minutes). Word boundaries were based on the manually corrected word annotations produced at MS State (Harkins et al., 2003). The word annotations allow measurement of word duration.

\subsection{Measuring Convergence}

There are several different approaches to measuring convergence. We adopt the method introduced by Cohen Priva et al. (2017) and Cohen Priva \& Sanker (2018). The benegits of this method are discussed by Cohen Priva \& Sanker (2019), but it has not yet been widely adopted, so we describe it here as well.

In shadowing tasks, speakers $(S)$ are exposed to a pre-recorded reference value $(R)$ and repeat after the recorded items produced by the model talker (e.g. Goldinger, 1998; Babel, 2012; Pardo et al., 2017). Speakers' characteristics are measured before the exposure $\left(S_{b}\right)$ and after the exposure to the model talker $\left(S_{R}\right)$. In conversational tasks, speakers' baseline values can be measured from the beginning of the conversation or in interactions with other interlocutors, as compared to their speech during or after conversing with the interlocutor. Convergence is a change in which the speaker becomes more like the model talker, i.e. a change from $S_{b}$ to $S_{R}$ that makes $S_{R}$ more like $R$. Following this conceptualization, Cohen Priva et al. (2017) model convergence using linear combination as in (1). The degree of convergence is measured as $\beta_{I}$, the relative importance of the interlocutor in predicting the performance of speakers after the interaction, relative to their consistency (self-correlation), which is measured as $\beta_{S_{b}}$. Thus, in this model, speakers' performance is predicted by a combination of their self-consistency, the performance of their interlocutor, and noise. Cohen Priva \& Sanker (2019) show that this method is not susceptible to artifacts caused by extreme initial values or the distance between the speaker and interlocutor, while measuring convergence with the commonly used difference-in-difference method produces artifacts of both. Earlier formulations of this model (Schweitzer \& Lewandowski, 2013) used a coefficient for the interlocutor alone, and modeled self-consistency $\beta_{S_{b}} S_{b}$ as a random intercept.

$$
S_{R} \approx \beta_{0}+\beta_{S_{b}} S_{b}+\beta_{R} R+\epsilon
$$


In this study, convergence is measured in two stages. First, each characteristic (e.g. F0) in each conversation is measured and processed separately. For every characteristic, all of the measurements for each speaker in each conversation are summarized to a single statistic (the median, inter-quartile range, or average, depending on the characteristic). Thus each conversation side is represented by a single value per characteristic. This step is illustrated for speech rate value in the top three and bottom three boxes in Figure 1.

Second, for each characteristic, each conversation side is summarized based on: (a) the speaker's performance during the conversation (equivalent to $S_{R}$ above), (b) the speaker's mean performance in other conversations, to be used as the speaker's baseline $S_{b}$, and (c) the interlocutor's mean performance in other conversations, to be used as the convergence target $(R)$.

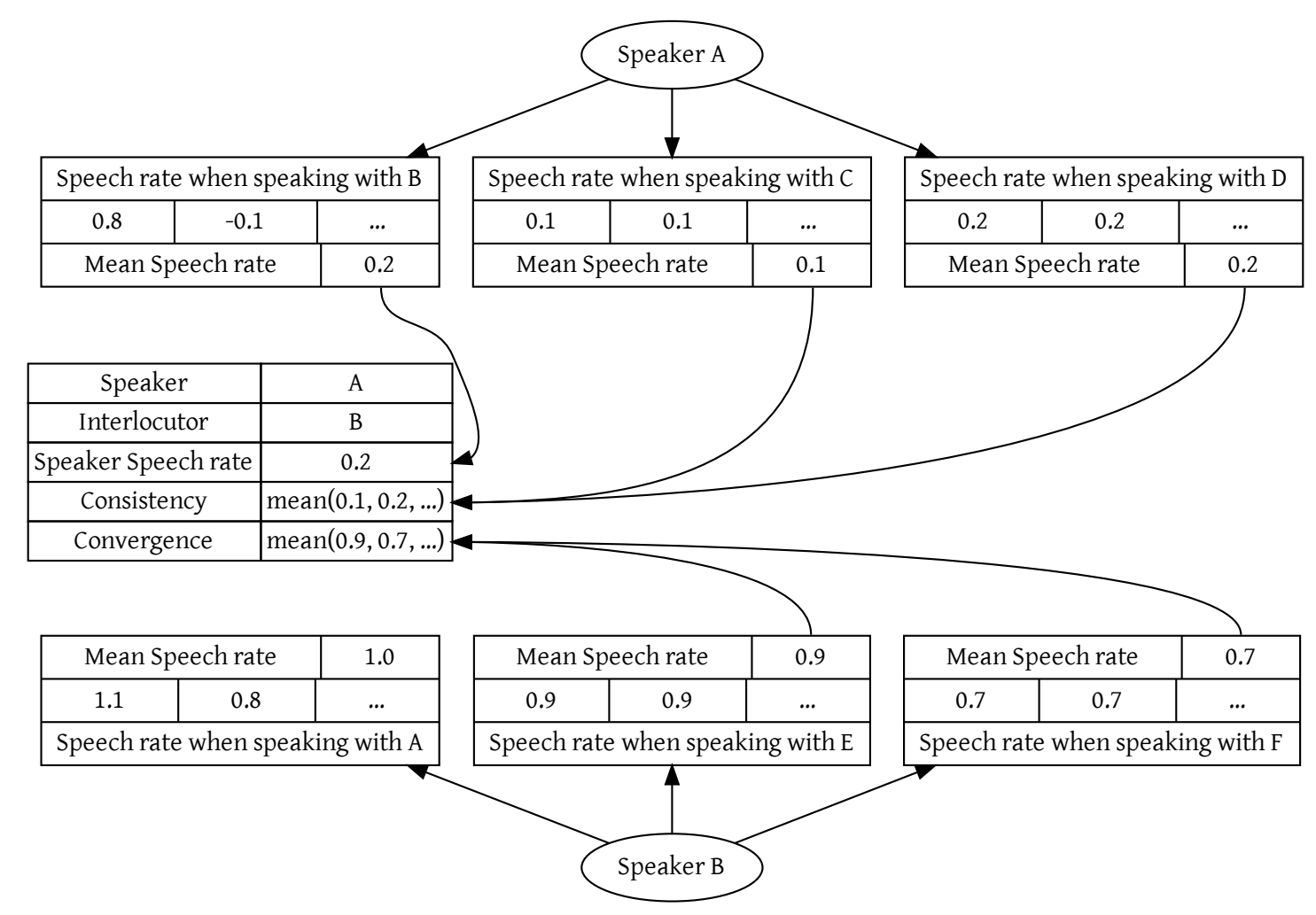

Figure 1: An illustration of the first two steps in the procedure, as they apply to speech rate. First (top three and bottom three boxes), the mean of multiple speech rate values (log observed over expected) is taken, yielding a single point per conversation side. Second, for each conversation side, the speaker's performance provides the predicted value, the mean of the speaker's performance in other conversations is used as the speaker's baseline $\left(S_{b}\right)$, and the interlocutor's performance in other conversations is averaged to yield the interlocutor's baseline $(R)$. 
Finally, these three data points for each characteristic are used to predict convergence, as illustrated for speech rate in Figure 2.

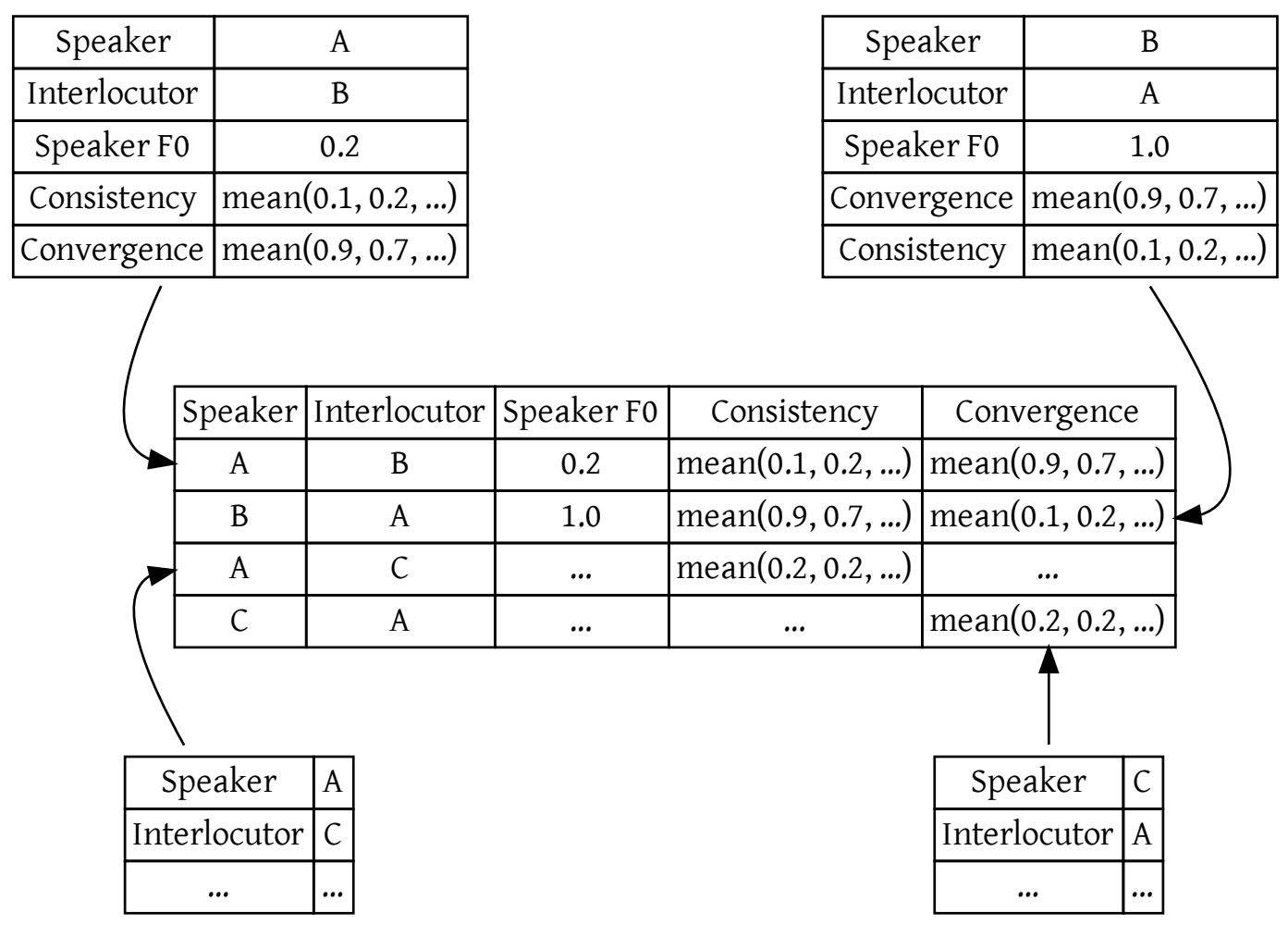

Figure 2: An illustration of the third and final step in the procedure, in which individual data points are combined to create the collection of data points for the analysis. Every conversation provides two summary data points, one for each side of the conversation.

The method measures convergence to the interlocutor's baseline, rather than to the interlocutor's performance in the shared conversation. This way of measuring convergence is necessary in order to exclude conversation-specific changes that may not be convergence per se (aspects of the environment or conversation that influence both speakers similarly), and to avoid attempting to estimate how a speaker's performance is influenced the interlocutor's performance, when both influence each other. While this method of using baselines from other conversations may decrease how much convergence is captured, convergence is still apparent when tested in this way. Establishing reliable baselines depends on having a large corpus, so that baselines are averaged across enough conversations to not be thrown off by any particular conversation; the median number of conversational partners for speakers in our dataset was 9 (Q1:4, Q3:12). 
The use of multiple data points per speaker and interlocutor necessitates adding random effects structure for repeated measures (Schweitzer \& Lewandowski, 2013; Cohen Priva \& Sanker, 2018). For example, random slopes for $\beta_{R}$ could be used to find individual differences in degree of convergence. If some speakers converge more than others, the model would fit those speakers with a somewhat higher $\beta_{R}$ than the population mean.

\subsection{Characteristics}

Convergence was measured in six characteristics. The goal was to have a broad range of characteristics. Four of the six, F0 median, F0 variance (IQR), speech rate, and uh:um ratio were examined by Cohen Priva \& Sanker (2018). Two characteristics were added to both expand the range of characteristics used and to include additional non-phonetic measures: lexical information rate and sentential conjunction. All six characteristics are described below.

F0 median F0 is the measure of the frequency of wave cycles produced by the vibration of the vocal folds. The measurement of frequency was converted into the mel scale, which provides a better approximation of human perception than $\mathrm{Hz}$ (Stevens et al., 1937).

F0 range F0 range was measured as the log of the ratio of the 75 th percentile to 25 th percentile of F0 measurements in mels.

Speech rate Speech rate in a conversation was measured as the mean log speech rate of individual utterances. Following Cohen Priva et al. (2017), point-wise speech rate was measured as the actual utterance duration (including pauses) divided by the expected utterance duration. Expected utterance duration was calculated as the sum of the predicted durations of words in the utterance, each calculated as the predicted value of a linear regression using the median duration of that word in the entire corpus, the length of the utterance, and the distance from the end of the utterance. Unlike F0 measurements, speech rate was calculated based on hand-corrected values.

uh:um ratio This measure was calculated as the log odds of $u h$ vs. um, two frequently-used filled pauses in English. The use of one or the other seems to be influenced by processing factors (e.g. Clark \& Fox Tree, 2002) as well as other factors such as gender (Acton, 2011). Log odds were calculated as the predicted values plus the residuals of a logistic regression between the number of $u h$ uses and $u m$ uses in each conversation side, which could be evaluated even when a subject never used one or the other.

Lexical information rate This measure was calculated following Cohen Priva (2017), as the mean negative log unigram predictability (the unigram entropy) of non-function words used in 
each conversation. Word counts were estimated using a combination of the Buckeye (Pitt et al., 2007), Fisher (Cieri et al., 2004, 2005), and Switchboard (Godfrey \& Holliman, 1997) corpora. Lexical information rate was chosen as it is easy to measure and average across a conversation (as opposed to tracking the use of individual words), and because it has been linked with speech rate (Cohen Priva, 2017), which increases the odds of finding common behaviors between the two measurs. Unigram lexical information rate captures the diversity and rarity of the words used by speakers. There are several ways that speakers may converge in lexical information rate. They may shift to more or less formal language registers or shift towards a shared specialized lexicon. To focus on such shifts, rather than on shifting choices in frequent words (e.g. expressions such as you know, that's right), we excluded the top $1 \%$ most frequent words. ${ }^{2}$

Sentential conjunction This characteristic measured the use of and to connect sentences, as in (2). In these cases, and is not strictly required, and the meaning of the sentence would change little if and were omitted, so sentences with and without this initial conjunction can be compared. These sentence-initial conjunctions have been described as discourse markers; they make clear the continuity between a statement and the preceding discourse and have various contextual predictors (Schiffrin, 1986; Dorgeloh, 2004).

For each conversation side, we counted the number of times "and PRON-NOM" appeared, where PRON-NOM could be any of $I$, she, he, or they ( 22,360 tokens). We excluded cases in which the preceding word was a given name ( 15 tokens), as in (3), sequences containing you and I ( 140 tokens), and word sequences of " $m y$ KINSHIP-TERM and I" ( 400 tokens), to exclude cases such as (4). Such constructions comprise the majority of "and PRON-NOM" sequences that are not cases of sentence conjunction. As with $u$ h:um ratio, we used a logistic regression to calculate the log odds between PRON-NOM with and without a preceding and, providing a comparison of pronominal sentences with or without sentential conjunction. There were 242,700 uses of PRON-NOM, of which $\sim 21,800$ were preceded by and.

(2) ...I certainly wouldn't object to it and I think random [testing for drugs] is probably, you know, the only really fair way ...(SW2638A)

(3) ...that Dan and I are going to ...(SW3323B)

(4) ...in the old days when my wife and I both worked ...(SW4238A)

As a preliminary step we verified that all six characteristics exhibit convergence. We therefore built mixed effects linear models following the formulation in (1), with random intercepts for

\footnotetext{
${ }^{2}$ Excluding the most frequent words does not have a substantial effect on the model results.
} 
the speaker, the interlocutor, the conversation, and the topic of conversation (as listed in the Switchboard corpus), as well as by-speaker and by-interlocutor random slopes for $\beta_{R}$. All the predictors and predicted values were standardized to allow easy comparison. This yields the formula provided in Table 1 (in lme4 syntax).

Speakers' baselines $\left(\beta_{S_{b}}\right)$ model speakers' self-consistency well, making by-speaker intercepts redundant. In five out of the six characteristics, the model fitted no variance to this by-speaker intercept. In the one remaining case, the by-speaker intercepts were fitted with values that were highly correlated (Pearson $r>.97$ ) with the $\beta_{S_{b}}$. We therefore refitted the models without by-speaker intercepts. The full data for the six studies, as well as the models in which by-speaker intercepts were not omitted, are provided in the supplementary materials.

Table 1:

\begin{tabular}{ll}
\hline lme4 syntax & explanation \\
\hline speaker & Speaker's performance in a conversation $\left(S_{R}\right)$ \\
1 & Intercept, expected to be zero $\left(\beta_{0}\right)$ \\
+ speaker.baseline & Speakers' baseline: captures consistency $\left(\beta_{S_{b}} S_{b}\right)$ \\
+ interlocutor.baseline & Interlocutors' baseline: captures convergence $\left(\beta_{R} R\right)$ \\
$+(1 \mid$ speaker $)$ & Differences by speaker \\
$+(1 \mid$ interlocutor $)$ & Differences by interlocutor \\
$+(1 \mid$ conversation $)$ & Differences by conversation \\
$+(1 \mid$ topic $)$ & Differences by topics \\
$+(0+$ interlocutor.baseline $\mid$ speaker $)$ & Per-speaker slope for convergence \\
$+(0+$ interlocutor.baseline $\mid$ interlocutor $)$ & Per-interlocutor slope for convergence \\
\hline
\end{tabular}

In every model, speakers were highly consistent in their production of that characteristic across conversations, as shown in Table 2, though consistency differed across the characteristics. Convergence, as indicated by the degree to which the interlocutor's baseline predicted for the variable, accounted for far less variance then the speaker's baseline, as Table 3 shows. However, significant convergence was found in every model.

Table 4 provides the standard deviation and model comparison-based $p$-values for the three random intercepts that remained in the model. Conversation significantly contributed to predicting the speaker's performance in every model. Because the conversation-level intercept applies to both conversation sides, it captures elements in the conversation in which the two participants vary together. This is not necessarily convergence, as it may be driven by external characteristics, rather than speakers shifting towards each other. Similarly, topic identity contributed to every model, indicating that different topics have different typical values (e.g. higher pitch, more $u h$ utterances). The interlocutor had a significant effect on speakers' performance only in the 
Table 2: Speaker consistency coefficients, per characteristic

\begin{tabular}{lrrrrr}
\hline & $\beta$ & SE & df & $t$ & $p$ \\
\hline F0 median & 0.971 & 0.0039 & 3434 & 248 & $<0.00001$ \\
F0 variance & 0.675 & 0.0119 & 3622 & 57 & $<0.00001$ \\
Speech rate & 0.800 & 0.0086 & 4336 & 93 & $<0.00001$ \\
uh:um ratio & 0.787 & 0.0090 & 4501 & 87 & $<0.00001$ \\
Lexical information rate & 0.645 & 0.0095 & 4490 & 68 & $<0.00001$ \\
Sentential conjunction & 0.391 & 0.0131 & 4677 & 30 & $<0.00001$ \\
\hline
\end{tabular}

Table 3: Convergence coefficients, per characteristic

\begin{tabular}{lrrrrr}
\hline & $\beta$ & SE & df & $t$ & $p$ \\
\hline F0 median & 0.0179 & 0.0049 & 181 & 3.7 & 0.00033 \\
F0 variance & 0.0929 & 0.0140 & 143 & 6.6 & $<0.00001$ \\
Speech rate & 0.0477 & 0.0097 & 219 & 4.9 & $<0.00001$ \\
uh:um ratio & 0.0320 & 0.0110 & 147 & 2.9 & 0.00433 \\
Lexical information rate & 0.0612 & 0.0110 & 224 & 5.5 & $<0.00001$ \\
Sentential conjunction & 0.0405 & 0.0148 & 152 & 2.7 & 0.00699 \\
\hline
\end{tabular}

speech rate model, though marginal effects were found for several of the other characteristics. The lack of consistent by-interlocutor intercepts suggests that the main influence that interlocutors have on speakers' performance is via convergence (measured with per-interlocutor slopes, and discussed below), rather than absolute effects.

Table 4: Random intercept SD and model-comparison $p$ values per characteristic

\begin{tabular}{lllllll}
\hline & \multicolumn{2}{c}{ Interlocutor } & \multicolumn{2}{c}{ Topic } & \multicolumn{2}{c}{ Conversation } \\
\hline & SD & $\mathrm{p}$ & $\mathrm{SD}$ & $\mathrm{p}$ & $\mathrm{SD}$ & $\mathrm{p}$ \\
F0 median & 0.036285 & 0.0680 & 0.0218 & 0.028 & 0.0743 & $<0.0001$ \\
F0 variance & 0.099476 & 0.0530 & 0.1227 & $<0.0001$ & 0.2602 & $<0.0001$ \\
Speech rate & 0.090704 & 0.0012 & 0.1288 & $<0.0001$ & 0.2943 & $<0.0001$ \\
uh:um ratio & 0.069665 & 0.1384 & 0.0509 & 0.024 & 0.2451 & $<0.0001$ \\
Lexical information rate & 0.000538 & 1.0000 & 0.4012 & $<0.0001$ & 0.2941 & $<0.0001$ \\
Sentential conjunction & 0.069681 & 0.4568 & 0.2145 & $<0.0001$ & 0.2241 & 0.0023 \\
\hline
\end{tabular}

Table 5 provides the standard deviation and model comparison-based $p$-values for the per-speaker and per-interlocutor random slopes for the interlocutors' baselines in the six models. These values indicate individual speakers' tendencies to converge and to elicit convergence, respectively. Per-speaker random slope was only significant for $u h$ :um convergence, and per-interlocutor ran- 
dom slope was only significant for lexical information rate. These results suggest that individual differences in convergence by speaker and by interlocutor, if they exist, are too weak to be reliably detected by the method used here. Recent work has generally also found a lack of consistency in the degree of convergence exhibited by individual speakers across tasks (Pardo et al., 2018) or in different conversations (Cohen Priva \& Sanker, 2018), though they exhibit some consistency within a characteristic in the same task (e.g. Tamminga et al., 2018). On the other hand, Pardo et al. (2017) found that some model talkers consistently elicited more convergence than others in the same shadowing task with the same recordings. Consistency across conversations with the same individual (e.g. Sanker, 2015) could reflect either individual tendencies in producing convergence or tendencies in the degree of convergence elicited by a particular individual.

Table 5: Random slope SD and model-comparison $p$ values per characteristic

\begin{tabular}{lllll}
\hline & \multicolumn{2}{l}{ Per-speaker convergence slope } & \multicolumn{2}{l}{ Per-interlocutor conversation slope } \\
\hline & $\mathrm{SD}$ & $\mathrm{p}$ & $\mathrm{SD}$ & $\mathrm{p}$ \\
F0 median & 0.0237 & 0.265 & 0.0297952809 & 0.22 \\
F0 variance & 0.0546 & 0.447 & 0.0607581348 & 0.32 \\
Speech rate & 0.0214 & 0.825 & 0.0000000928 & 1.00 \\
uh:um ratio & 0.0734 & 0.017 & 0.0436640104 & 0.44 \\
Lexical information rate & 0.0000 & 1.000 & 0.0797569270 & 0.02 \\
Sentential conjunction & 0.0637 & 0.326 & 0.0628545526 & 0.38 \\
\hline
\end{tabular}

\section{Meta-analysis across characteristics}

\subsection{Introduction}

While the by-characteristic analysis confirmed that convergence exists in all of the characteristics measured, there was only weak evidence for individual tendencies in producing convergence or eliciting convergence. These models also could not detect convergence patterns particular to each conversation, as each conversation has only two summary data points per characteristic (one for each speaker). In order to evaluate by-speaker, by-interlocutor, and by-conversation patterns in convergence as reflected across characteristics, we we grouped together all six characteristics (they were already standardized, to facilitate comparison).

Previous studies have found individual differences in various perceptual tasks (e.g. Johnson et al., 1987; Kong \& Edwards, 2016; Ishida et al., 2016), and some work has found that personal characteristics such as Autism Quotient (Yu et al., 2013) and social desirability scores (Natale, 1975) are 
predictive of degree of convergence, but studies on individual differences in convergence have rarely used retesting to establish individual consistency in convergence. Individual tendencies in convergence may exist but be outweighed by larger effects such as the nature of each particular conversation or the speaker's opinion of the interlocutor. In this case, convergent patterns by conversation or by interlocutor may be more apparent than convergent tendencies of individuals. Such findings would support accounts of social factors mediating convergence (e.g. Giles et al., 1973; Babel, 2009; Pardo et al., 2012).

\subsection{Statistical Models}

The six models in the by-characteristic analysis presented in the previous section each have a mixed effects model predicting the performance of the speakers based on their own baselines and the baselines of their interlocutors along a single characteristic. The meta-analysis presented here tests convergence across all characteristics. This provides up to 12 summary data points per conversation (6 characteristics for each speaker); not all characteristics were available for all conversations, given exclusion procedures based on sound quality and the usage of each characteristic. As described above, each data point is a summary of the speaker's behavior in that characteristic across the entire conversation.

The regression analysis used each speaker's performance in a conversation as the dependent variable (once for each characteristic). The speaker's baseline in other conversations and the interlocutor's baseline in other conversations were used as the two main fixed predictors. The interlocutor's baseline in other conversations is the variable that measures convergence: A stronger effect of the interlocutor's baseline as a predictor of the speaker's productions indicates more convergence. These methods are the same used for the six individual characteristics in the bycharacteristic analysis, except that each speaker in a conversation is repeated for every characteristic. The main difference is in the structure of the random effects. The elements of the model are outlined below.

Several of the factors treat the characteristics as a pooled group; these factors are referred to as characteristic-agnostic, in contrast to the factors which test differences across characteristics, which are per-characteristic.

Several of the possible random intercepts would be meaningless in the context of the current study. For instance, it is meaningful to expect per-interlocutor intercepts to differ within each characteristic, but characteristic-agnostic per-interlocutor intercepts would arbitrarily group together different characteristics (e.g. higher F0 and more use of $u h$ ). In contrast, characteristic- 
agnostic random slopes for convergence abstract over a putative single behavior, "participating in convergence," and should therefore be included in the model. The modeling choices are listed below, and summarized in Table 6.

Characteristic The model contains the per-characteristic random slopes, one for the speaker's baseline and one for the interlocutor's baseline. The two slopes are meant to account for the differences in consistency and convergence in different characteristics, as observed in the by-characteristic analysis. A per-characteristic intercept was not included, because the intercept was not significantly different than zero in the individual models in the bycharacteristic analysis.

Conversation We included a characteristic-agnostic random slope for convergence, and a percharacteristic random intercept. We did not include a characteristic-agnostic random intercept, as explained above. Within-characteristic intercepts are meant to explain the variance captured by per-conversation intercepts in the by-characteristic analysis 1 . The random slope per conversation is one of four main variables of interest: If particular conversations elicit a higher or lower degree of convergence across multiple characteristics, this variable would significantly contribute to the model.

Speaker Despite its ultimate exclusion in the models for the individual characteristics, we included a within-characteristic random intercept per speaker. We also included a withincharacteristic per speaker random slope for the interlocutors' baseline to model withincharacteristic individual differences in convergence, as well as a characteristic-agnostic random slope for the interlocutors' baseline to model an overall tendency to converge. If there are individual tendencies in producing convergence, such that some speakers consistently converge more than other speakers ("followers"), the slope would contribute to the model, making it better than a minimally different model that does not contain that slope. This slope is the second main variable of interest.

Interlocutor Interlocutor factors parallel the per-speaker random structure. We included a within-characteristic random intercept per-interlocutor, which mirrors the per-interlocutor random intercepts in the Study 1 models. We also included both a within-characteristic and characteristic-agnostic random slopes for convergence. The former mirrors the per-interlocutor random slope for convergence in the studies for individual characteristics, and the latter models the possibility that there are general tendencies in how much convergence particular interlocutors elicit, such that some interlocutors elicit more convergence than other interlocutors ("leaders"), across different speech characteristics. This is the third main variable of interest. 
Topic For topic, we used a within-characteristic random intercept, which mirrors the per-topic intercept in the by-characteristic models.

Table 6: The lme4 formula used to fit the model in the meta-analysis

\begin{tabular}{ll}
\hline lme4 syntax & explanation \\
\hline performance $~$ & Speaker's performance in a conversation \\
1 & Global intercept (expected to be 0$)$ \\
+ speaker.baseline & Speaker's baseline performance for the characteristic \\
+ interlocutor.baseline & Interlocutor's baseline performance for the characteristic \\
$+(0+$ speaker.baseline $\mid$ char $)$ & Per-characteristic slope for consistency \\
$+(0+$ interlocutor.baseline $\mid$ char $)$ & Per-characteristic slope for convergence \\
$+(0+$ interlocutor.baseline | char:speaker $)$ & Within-characteristic per-speaker slope for convergence \\
$+(0+$ interlocutor.baseline $\mid$ char:interlocutor $)$ & Within-characteristic per-interlocutor slope for convergence \\
$+(1 \mid$ char:topic $)$ & Within-characteristic per-topic differences \\
$+(1 \mid$ char:speaker $)$ & Within-characteristic per-speaker differences \\
$+(1 \mid$ char:interlocutor $)$ & Within-characteristic per-interlocutor differences \\
$+(1 \mid$ char:conversation $)$ & Within-characteristic per-conversation differences \\
$+(0+$ interlocutor.baseline $\mid$ speaker $)$ & Per-speaker slope for convergence (characteristic agnostic) \\
$+(0+$ interlocutor.baseline $\mid$ interlocutor $)$ & Per-interlocutor slope for convergence (characteristic agnostic \\
$+(0+$ interlocutor.baseline $\mid$ conversation $)$ & Per-conversation slope for convergence (characteristic agnost \\
\hline
\end{tabular}

\subsection{Results and discussion}

As expected, both the speakers' own baseline and their interlocutors' baseline performance were significant predictors of the speakers' performance $(\beta=0.711, \mathrm{SE}=0.079, \mathrm{df}=5, t=8.95, p=0.00029$; $\beta=0.0471, \mathrm{SE}=0.011, \mathrm{df}=5, t=4.45, p=0.00596$, respectively): speakers' exhibited self-consistency in their productions across conversations, and they exhibited convergence. The results for the random effects are summarized in Table 7.

Among the variables of interest, only the per-interlocutor slope for convergence significantly improved the model $(\mathrm{SD}=0.0454 p=0.0016)$. We found no evidence for a per-speaker slope for convergence ( $\mathrm{SD}=0 p=1)$, suggesting that the effect of "natural leaders" in convergence is easier to find than the effect of "natural followers." Similarly, we found no evidence for a per-conversation slope for convergence $(\mathrm{SD}=0.0000315 p=1)$.

As expected, there were significant differences in per-characteristic slope for consistency and per-characteristic slope for convergence $(\mathrm{SD}=0.194 p=<0.0001 ; \mathrm{SD}=0.0227 p=0.0012$, respectively); both self-consistency and convergence varied by characteristic. Similarly, as in 
Table 7: Summary of the meta-analysis random effcts

\begin{tabular}{lll}
\hline & SD & Model comparison $p$ \\
\hline Per-characteristic and conversation intercept & 0.2473518 & $<0.0001$ \\
Per-characteristic and interlocutor slope for convergence & 0.0143233 & 0.86616 \\
Per-characteristic and interlocutor intercept & 0.0714960 & 0.00053 \\
Per-characteristic and speaker slope for convergence & 0.0459992 & 0.04416 \\
Per-characteristic and speaker intercept & 0.0000000 & 1.00000 \\
Conversation slope for convergence & 0.0000315 & 1.00000 \\
Interlocutor slope for convergence & 0.0453861 & 0.00160 \\
Speaker slope for convergence & 0.0000000 & 1.00000 \\
Per-characteristic and topic intercept & 0.2010929 & $<0.0001$ \\
Per-characteristic slope for convergence & 0.0226591 & 0.00123 \\
Per-characteristic slope for consistency & 0.1942646 & $<0.0001$ \\
\hline
\end{tabular}

the per-characteristic models, there were within-characteristic per-conversation differences and within-characteristic per-topic differences $(\mathrm{SD}=0.247 p=<0.0001 ; \mathrm{SD}=0.201 p=<0.0001)$; there were absolute patterns in how each characteristic was realized based on the particular conversation and based on the conversation topic.

Unlike the per-characteristic models, the within-characteristic per-interlocutor differences did improve the model ( $\mathrm{SD}=0.0715 p=0.00053)$, as did the within-characteristic per-speaker slope for convergence $(\mathrm{SD}=0.046 p=0.044)$. That is, there were by-speaker and by-interlocutor tendencies in convergence that were specific to the particular characteristic being measured. The differences between these factors in this combined model and in the models for individual characteristics could have arisen because the modeling of the fixed effects is not identical across characteristics. No other random effect had a significant effect on the model.

Although there was only a weak per-interlocutor effect in one of the models for convergence in individual characteristics, there was a significant per-interlocutor effect in this meta-study. The combined study makes it possible to identify interlocutor effects that are definitely convergence (per-interlocutor slope for interlocutor performance as a predictor of the speakers' performance), rather than particular interlocutors motivating an absolute change in a particular measure (perinterlocutor intercept). In studies for individual speech characteristics, it can be difficult to distinguish between these factors, as both might produce the same effects. However, when comparing across speech characteristics, it will be clear if the shift elicited by the interlocutor is convergent or merely an absolute effect in a particular characteristic. The influence of the interlocutor on degree of convergence across characteristics provides support for convergence being socially 
mediated at a high level.

Some of the per-interlocutor consistency can be explained by the social status or likeability of the interlocutor; speakers converge more to more socially desirable interlocutors (Gregory \& Webster, 1996; Hwang \& Chun, 2018) and to interlocutors whom they like more (Yu et al., 2011; Sanker, 2015). Our study does not aim to identify the particular characteristics that make some interlocutors elicit more convergence than others. However, Switchboard does provide demographic information about the speakers, so we did a post-hoc test of whether any of these factors predicted the per-interlocutor variation in convergence. None of the factors were significant (for this model, see the Supplementary Materials, Section 3), though there was a marginal tendency for older speakers to elicit less convergence. This model also tested whether amount of speech produced by the interlocutor, either counted by word or by time, predicted per-interlocutor convergence; neither factor was a significant predictor of how much convergence each interlocutor elicited, indicating that per-interlocutor variation in convergence is not due to variation in talkativeness and consequently amount of exposure.

In contrast to the per-interlocutor slope, the per-speaker convergence slope did not improve the model. Given that the method was sensitive enough to find support for per-interlocutor tendencies in convergence, this null result is not likely to be due to insufficient power. Individual propensity to converge was not strong enough for our model to detect, providing little support for the view of convergence as reflecting inherent individual traits. The results instead support the argument that variation in degree of convergence largely reflects properties of the interaction rather than aspects of the individual. Notably, per-interlocutor convergence is not a form of individual differences in the sense of individual tendencies in production or perception. Interlocutors who elicit greater convergence have characteristics that make others converge to them; they don't have internal tendencies in producing convergence themselves.

\section{General Discussion}

Our paper addresses two main questions: Is there evidence for individual tendencies in convergence, reflected similarly across characteristics, which would indicate a broad cognitive trait responsible for convergence? Is there evidence for consistent effects of particular interlocutors in eliciting convergence, which would indicate social effects in mediating convergence? Both of these issues fit into a larger question of how convergence arises and what drives variation in convergence. 


\subsection{Speaker Effects in Convergence}

Within our data, we found no evidence for a contribution of individual tendencies in predicting convergence across characteristics. On the other hand, the interlocutor was a significant predictor of degree of convergence in our data, with exactly the same sample size as the speaker, as the same individuals were considered in both roles; thus, the lack of evidence for individual tendencies in convergence is unlikely to result from inadequate sample size.

Individual differences in perception have been demonstrated previously, and can have high testretest reliability with the same or closely related tests, while correlations across less closely related tests tend to be weak and the differences do not correlate well with performance in most cognitive tests (e.g. Surprenant \& Watson, 2001; Kidd et al., 2007). There is more limited evidence for individual consistency in convergence. Some characteristics of the speaker are predictive of degree of convergence, for example, Autism Quotient (Yu et al., 2013), social desirability scores (Natale, 1975), social network size (Lev-Ari, 2018), and tendency to compromise (Weatherholtz et al., 2014). However, few convergence studies include re-testing to demonstrate that individuals are consistent in these differences, and the size of the effect is often small. There is some evidence for individual tendencies in convergence within a given characteristic between instances of same task or similar tasks (Tamminga et al., 2018; Sanker, 2015), but evidence for tendencies across different tasks is weaker (Pardo et al., 2018). The results of our study, in which each individual conversed with several different interlocutors on different topics, provide little evidence for individual tendencies in convergence.

While some individual tendencies in convergence may exist, our results suggest that they do not reflect any broad cognitive trait (cf. Yu, 2013; Yu et al., 2013), but rather must be specific to particular characteristics. Little previous work has tested correlations across characteristics; the work that has looked for them has generally found no correlations (Bilous \& Krauss, 1988; Pardo et al., 2012; Sanker, 2015; Weise \& Levitan, 2018), with the occasional correlation that can probably be attributed to physical relationships between characteristics (Cohen Priva \& Sanker, 2018) or repeated measures producing a false positive (Sanker, 2015; Rahimi et al., 2017). In studies with a relatively small number of participants, a null result for individual tendencies can be difficult to interpret; there has been no previous large-scale comparison across characteristics, controlling for effects of particular conversations. Our study, using a corpus with a large number of speakers, provides clearer evidence for a lack of individual convergent tendencies that hold across characteristics. The absence of a significant effect in this large corpus indicates that if broad individual differences in convergence do exist, they would be too weak to be detected in normal experimental settings. 


\subsection{Interlocutor Effects in Convergence}

The results of our study indicate that variation in convergence is influenced by the interlocutor: Individual interlocutors have tendencies in how much convergence they elicit. Previous studies with smaller sets of individuals have similarly demonstrated variation based on the interlocutor or model talker (e.g. Pardo et al., 2017; Hwang \& Chun, 2018), and effects of particular manipulations of the actual or perceived characteristics of the interlocutor or model talker, such as interlocutor status (e.g. Gregory \& Webster, 1996; Bane et al., 2010) and the speaker's opinion of the interlocutor or model talker (e.g. Babel, 2010; Yu et al., 2011). Our finding of interlocutor-specific variation in convergence is consistent across characteristics; that is, the factors that strengthen or weaken convergence to a particular interlocutor in one linguistic characteristic similarly affect other characteristics.

The presence of strong interlocutor influences within our data suggests that convergence is socially mediated, even if the mechanism driving convergence is not inherently social. Speakers converge more to speakers whom they like based either on the context of their interaction (Babel, 2010; Yu et al., 2011) or whom they perceive as high status (Gregory \& Webster, 1996; Bane et al., 2010), and they are also more likely to converge to voices that are perceived as more standard (Weatherholtz et al., 2014) or more attractive (Babel et al., 2014). By-interlocutor consistency in convergence even across different linguistic characteristics and across conversations with different partners and different conversational topics suggests that the social effects occur at a high level, perhaps reflecting interspeaker alignment (cf. Pickering \& Garrod, 2004; Jiang et al., 2015), with different interlocutors eliciting different degrees of alignment.

Variation in convergence elicited by each interlocutor cannot be attributed to how much exposure speakers received to the interlocutor. Within our data, per-interlocutor convergence was not correlated with the average amount of speech produced by that interlocutor, either as measured in number of words or total time spent talking. Consistent with this lack of correlation, variation in convergence elicited by different interlocutors has also been observed in previous studies which provided equal exposure to each voice (e.g. Pardo et al., 2017; Hwang \& Chun, 2018).

\subsection{Conversation Effects in Convergence}

In contrast with interlocutor effects, the particular conversation was not a significant predictor of convergence. Some previous work has found that degree of convergence is predicted by aspects of the particular relationship between interlocutors (e.g. Pardo et al., 2012; Bane et al., 2010; Sanker, 2015). However, such work has often looked at each participant in a single interaction, 
so effects of the interlocutors and effects of the particular conversation could not be separated out, except indirectly based on finding differences associated with characteristics of the speakers or the conversation, or experimental conditions manipulating aspects of the conversation. While our results do not provide evidence for effects of the conversation, we have less data for each conversation than for each speaker or interlocutor, so the lack of per-conversation effect in our results might simply be the result of insufficient data to capture such an effect.

\section{Conclusion}

We present new data on convergence across characteristics and across speakers, using a large corpus of natural speech. Although there is variation across characteristics in convergence and in speakers' consistency, different characteristics exhibited some shared influences, which provides

evidence for high-level mediation of convergence, reflected across different linguistic characteristics.

There was little evidence for speaker tendencies in how much they converged; to the extent that individual tendencies in convergence exist, they are specific to particular characteristics, rather than reflecting a broad cognitive trait. Thus, individual tendencies in convergence are unlikely to provide strong insights into individual linguistic variation in broad traits, but might inform variation in behavior of specific details.

On the other hand, the interlocutor was predictive of degree of convergence across characteristics and across conversations, suggesting that there might be "leaders" whose speech listeners are more likely to imitate. These results indicate the importance of situational factors in mediating convergence and moreover suggest that this mediation occurs at a high level.

\section{References}

Abel, J. \& Babel, M. (2017). Cognitive load reduces perceived linguistic convergence between dyads. Language and Speech, 60(3), 479-502.

Acton, E. K. (2011). On gender differences in the distribution of um and uh. In University of Pennsylvania Working Papers in Linguistics, volume 17 chapter 2. https://repository.upenn.edu /pwpl/vol17/iss2/2.

Babel, M. (2009). Phonetic and Social Selectivity in Speech Accommodation. PhD thesis, University of California, Berkeley. 
Babel, M. (2010). Dialect divergence and convergence in New Zealand English. Language in Society, 39, 437-456.

Babel, M. (2012). Evidence for phonetic and social selectivity in spontaneous phonetic imitation. Journal of Phonetics, 40(1), 177-189.

Babel, M., McGuire, G., Walters, S., \& Nicholls, A. (2014). Novelty and social preference in phonetic accommodation. Laboratory Phonology, 5(1), 123-150.

Bane, M., Graff, P., \& Sonderegger, M. (2010). Longitudinal phonetic variation in a closed system. In Proceedings from the Annual Meeting of the Chicago Linguistic Society, volume 46 (pp. 43-58).: Chicago Linguistic Society.

Bilous, F. R. \& Krauss, R. M. (1988). Dominance and accommodation in the conversational behaviours of same-and mixed-gender dyads. Language \& Communication, 8(3), 183-194.

Chartrand, T. L. \& Bargh, J. A. (1999). The chameleon effect: The perception-behavior link and social interaction. Journal of Personality and Social Psychology, 76(6), 893-910.

Cieri, C., Graff, D., Kimball, O., Miller, D., \& Walker, K. (2005). Fisher English training part 2, transcripts. Linguistic Data Consortium, Philadelphia.

Cieri, C., Miller, D., \& Walker, K. (2004). The Fisher corpus: A resource for the next generations of speech-to-text. In Proceedings of the 4th International Conference on Language Resources and Evaluation (pp. 69-71).

Clark, H. H. \& Fox Tree, J. E. (2002). Using uh and um in spontaneous speaking. Cognition, 84(1), 73-111.

Cohen Priva, U. (2017). Not so fast: Fast speech correlates with lower lexical and structural information. Cognition, 160, 27-34.

Cohen Priva, U., Edelist, L., \& Gleason, E. (2017). Converging to the baseline: Corpus evidence for convergence in speech rate to interlocutor's baseline. Fournal of the Acoustical Society of America, 141(5), 2989-2996.

Cohen Priva, U. \& Sanker, C. (2018). Distinct behaviors in convergence across measures. In Proceedings of the 40th Annual Meeting of the Cognitive Science Society (pp. 1515-1520).

Cohen Priva, U. \& Sanker, C. (2019). Limitations of difference-in-difference for measuring convergence. Laboratory Phonology: Journal of the Association for Laboratory Phonology, 10(1), 15. 
Dorgeloh, H. (2004). Conjunction in sentence and discourse: Sentence-initial and and discourse structure. Journal of Pragmatics, 36(10), 1761-1779.

Gijssels, T., Casasanto, L. S., Jasmin, K., Hagoort, P., \& Casasanto, D. (2016). Speech accommodation without priming: The case of pitch. Discourse Processes, 53(4), 233-251.

Giles, H., Coupland, N., \& Coupland, J. (1991). Accommodation theory: Communication, context, and consequence. In H. Giles, J. Coupland, \& N. Coupland (Eds.), Contexts of Accommodation: Developments in Applied Sociolinguistics (pp. 1-68). New York: Cambridge University Press.

Giles, H., Taylor, D., \& Bourhis, R. (1973). Towards a theory of interpersonal accommodation through language: Some Canadian data. Language in Society, 2, 177-192.

Godfrey, J. J. \& Holliman, E. (1997). Switchboard-1 release 2. Linguistic Data Consortium, Philadelphia.

Goldinger, S. D. (1998). Echoes of echoes? An episodic theory of lexical access. Psychological Review, 105(2), 251-279.

Gregory, S. W. J. \& Webster, S. (1996). A nonverbal signal in voices of interview partners effectively predicts communication accommodation and social status perceptions. Fournal of Personality and Social Psychology, 70(6), 1231-1240.

Harkins, D., Feinstein, D., Lindsey, T., Martin, S., \& Winter, G. (2003). Switchboard MS State manually corrected word alignments. https:/www.isip.piconepress.com/projects/switchboar $\mathrm{d} /$.

Heath, J. (2017). How automatic is phonetic convergence? Evidence from working memory. In Proceedings of the Linguistic Society of America, volume 2 (pp. Art. 35).

Hwang, H. \& Chun, E. (2018). Influence of social perception and social monitoring on structural priming. Cognitive Science, 42, 303-313.

Ishida, M., Samuel, A. G., \& Arai, T. (2016). Some people are "more lexical" than others. Cognition, $151,68-75$.

Jiang, J., Chen, C., Dai, B., Shi, G., Ding, G., Liu, L., \& Lu, C. (2015). Leader emergence through interpersonal neural synchronization. PNAS, 112(14), 4274-4279.

Johnson, D. M., Watson, C. S., \& Jensen, J. K. (1987). Individual differences in auditory capabilities. I. Fournal of the Acoustical Society of America, 81(2), 427-438.

Kaschak, M. P., Kutta, T. J., \& Jones, J. L. (2011). Structural priming as implicit learning: Cumu- 
lative priming effects and individual differences. Psychonomic Bulletin \& Review, 18(6), 11331139.

Kidd, G. R., Watson, C. S., \& Gygi, B. (2007). Individual differences in auditory abilities. Fournal of the Acoustical Society of America, 122(1), 418-435.

Kim, M., Horton, W. S., \& Bradlow, A. R. (2011). Phonetic convergence in spontaneous conversations as a function of interlocutor language distance. Laboratory Phonology, 2(1), 125-156.

Kong, E. J. \& Edwards, J. (2016). Individual differences in categorical perception of speech: Cue weighting and executive function. Journal of Phonetics, 59, 40-57.

Lev-Ari, S. (2018). Social network size can influence linguistic malleability and the propagation of linguistic change. Cognition, 176, 31-39.

Levitan, R. \& Hirschberg, J. B. (2011). Measuring acoustic-prosodic entrainment with respect to multiple levels and dimensions. In Proceedings of Interspeech (pp. 3081-3084). Brisbane: International Speech Communications Association.

Namy, L. L., Nygaard, L. C., \& Sauerteig, D. (2002). Gender differences in vocal accommodation: The role of perception. Journal of Language and Social Psychology, 21(4), 422-432.

Natale, M. (1975). Convergence of mean vocal intensity in dyadic communication as a function of social desirability. Journal of Personality and Social Psychology, 32(5), 790-804.

Oben, B. \& Brône, G. (2016). Explaining interactive alignment: A multimodal and multifactorial account. Fournal of Pragmatics, 104, 32-51.

Pardo, J. S., Gibbons, R., Suppes, A., \& Krauss, R. M. (2012). Phonetic convergence in college roommates. Journal of Phonetics, 40, 190-197.

Pardo, J. S., Urmanche, A., Wilman, S., \& Wiener, J. (2017). Phonetic convergence across multiple measures and model talkers. Attention, Perception, \& Psychophysics, 79, 637-659.

Pardo, J. S., Urmanche, A., Wilman, S., Wiener, J., Mason, N., Francis, K., \& Ward, M. (2018). A comparison of phonetic convergence in conversational interaction and speech shadowing. fournal of Phonetics, 69, 1-11.

Pickering, M. J. \& Garrod, S. (2004). Towards a mechanistic psychology of dialogue. Behavioral and Brain Sciences, 27, 169-190.

Pickering, M. J. \& Garrod, S. (2013). An integrated theory of language production and comprehension. Behavioral and Brain Sciences, 36, 329-392. 
Pitt, M., Dilley, L., Johnson, K., Kiesling, S., Raymond, W., Hume, E., \& Fosler-Lussier, E. (2007). Buckeye corpus of conversational speech (2nd release). Department of Psychology, Ohio State University.

Rahimi, Z., Kumar, A., Litman, D., Paletz, S., \& Yu, M. (2017). Entrainment in multi-party spoken dialogues at multiple linguistic levels. In Proceedings of Interspeech (pp. 1696-1700). Stockholm, Sweden.

Sanker, C. (2015). Comparison of phonetic convergence in multiple measures. In Cornell Working Papers in Phonetics and Phonology 2015 (pp. 60-75).

Schiffrin, D. (1986). Functions of and in discourse. Journal of Pragmatics, 10, 41-66.

Schweitzer, A. \& Lewandowski, N. (2013). Convergence of articulation rate in spontaneous speech. In Proceedings of Interspeech (pp. 525-529).

Stevens, S., Volkmann, J., \& Newman, E. (1937). A scale for the measurement of psychological magnitude pitch. Journal of the Acoustical Society of America, 8, 185-190.

Surprenant, A. M. \& Watson, C. S. (2001). Individual differences in the processing of speech and nonspeech sounds by normal-hearing listeners. Journal of the Acoustical Society of America, 110(4), 2085-2095.

Tamminga, M., Wade, L. A., \& Lai, W. (2018). Stability and variability in phonetic flexibility. In 92nd Annual Meeting of the Linguistic Society of America Salt Lake City, Utah.

Weatherholtz, K., Campbell-Kibler, K., \& Jaeger, T. F. (2014). Socially mediated syntactic alignment. Language Variation and Change, 26, 387-420.

Weise, A. \& Levitan, R. (2018). Looking for structure in lexical and acoustic-prosodic entrainment behaviors. In Proceedings of the 2018 Conference of the North American Chapter of the Association for Computational Linguistics: Human Language Technologies, volume 2 (pp. 297-302).

$\mathrm{Yu}, \mathrm{A}$. (2013). Individual differences in socio-cognitive processing and the actuation of sound change. In A. Yu (Ed.), Origins of Sound Change: Approaches to Phonologization (pp. 201-227). Oxford, England: Oxford University Press.

Yu, A., Abrego-Collier, C., Baglini, R., Grano, T., Martinovic, M., Otte III, C., Thomas, J., \& Urban, J. (2011). Speaker attitude and sexual orientation affect phonetic imitation. University of Pennsylvania Working Papers in Linguistics, 17(1), 235-242.

Yu, A., Abrego-Collier, C., \& Sonderegger, M. (2013). Phonetic imitation from an individual- 
difference perspective: Subjective attitude, personality and "autistic" traits. PloS ONE, 8(9), e74746.

Yun, K., Watanabe, K., \& Shimojo, S. (2012). Interpersonal body and neural synchronization as a marker of implicit social interaction. Scientific Reports, 2, Art. 959. 